\title{
Des enfants-médiateurs et leur perception des objets et des personnages historiques d'un musée d'histoire au Québec
}

\section{Thérèse Martin, Université de Bordeaux 3}

therese.martin@wanadoo.fr

Résumé Dans nos recherches précédentes (Martin, 2011), nous nous étions intéressés à l'expérience de visite des enfants (de 7 à 11 ans) dans les musées de sciences. À présent, nous portons notre intérêt à l'expérience de visite dans les musées d'histoire. Cette recherche exploratoire portant sur un petit groupe d'enfants-«guides » vise à mettre en évidence les relations établies par des enfants avec les objets du musée et les personnages historiques exposés. Les paroles des enfants ainsi recueillies lors de leur présentation de l'exposition (ou dans leur rôle de «guide ») nous permettront de rendre compte des différents modes d'approches de l'exposition du Musée des Ursulines à Québec.

Mots clés: Musée d'histoire et de civilization; visiteurs-enfants; expérience de visite; interpretation; médiation du patrimoine.

\section{Questionnement de recherche: quelle (s) expérience (s) de visite vécue (s) par les enfants « guides »?}

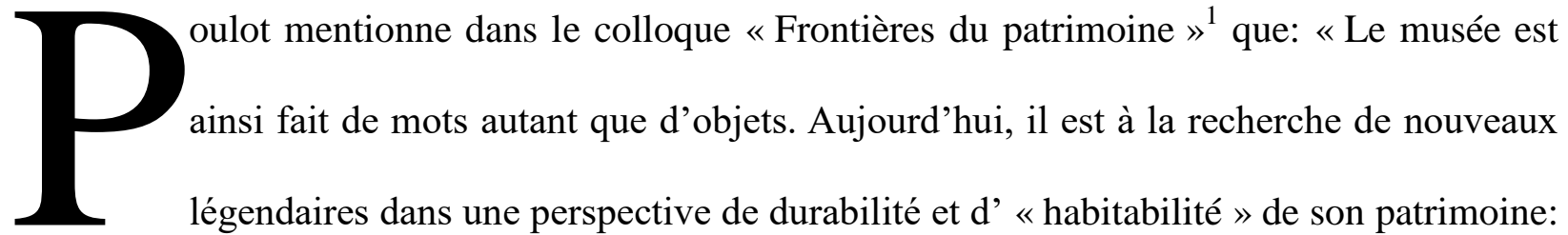

c'est évoquer la désirabilité de son expérience et le bien-être de ses visiteurs ». Sous cet éclairage, nous portons intérêt aux visiteurs-enfants dans les musées d'histoire et à leurs relations au patrimoine.

Cette recherche s'inscrit dans le cadre de l'exploration des manières dont des enfants (âgés de 7 à 11 ans) perçoivent les objets et les personnages historiques présentés dans des expositions de musées d'histoire et autres lieux de transmission du patrimoine au Québec. Cela permet ainsi de dégager leur interprétation et leur mode d'appropriation du patrimoine historique. Cette

\footnotetext{
${ }^{1}$ Colloque « Frontières du patrimoine, circulation des savoirs, des objets et œuvres d'art », 27-28 mars 2015 à Paris. https://patrimoine.hypotheses.org/date/2015/03
} 
recherche exploratoire a lieu au musée des Ursulines à Québec. Elle fait suite à une précédente recherche réalisée dans le contexte des institutions muséales de sciences et qui visait à rendre compte de l'expérience de visite des enfants dans l'espace de médiation de l'exposition.

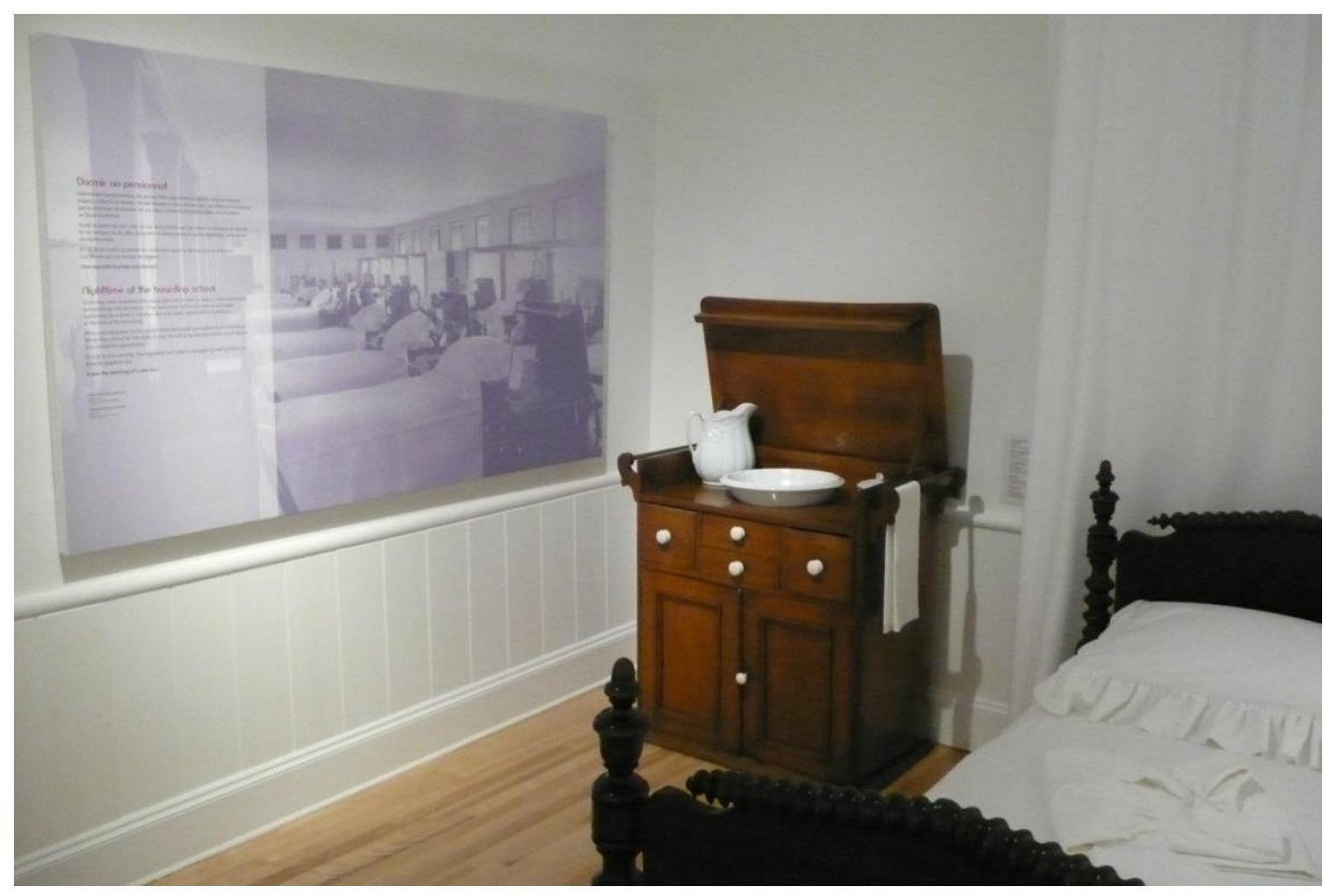

Fig. 1: Entrée de la salle « au son de la cloche »

\section{Recueil des paroles des enfants et terrain de recherche}

La méthode consiste à proposer aux enfants de présenter une des salles de musée. Dans un premier temps, ce sont des enfants (six) de l'école des Ursulines qui ont été "guides » sur le temps des loisirs. Ultérieurement, ce seront des enfants en visite dans le cadre des loisirs (en famille) qui seront sollicités pour cette mise en situation de rôle de guide. 


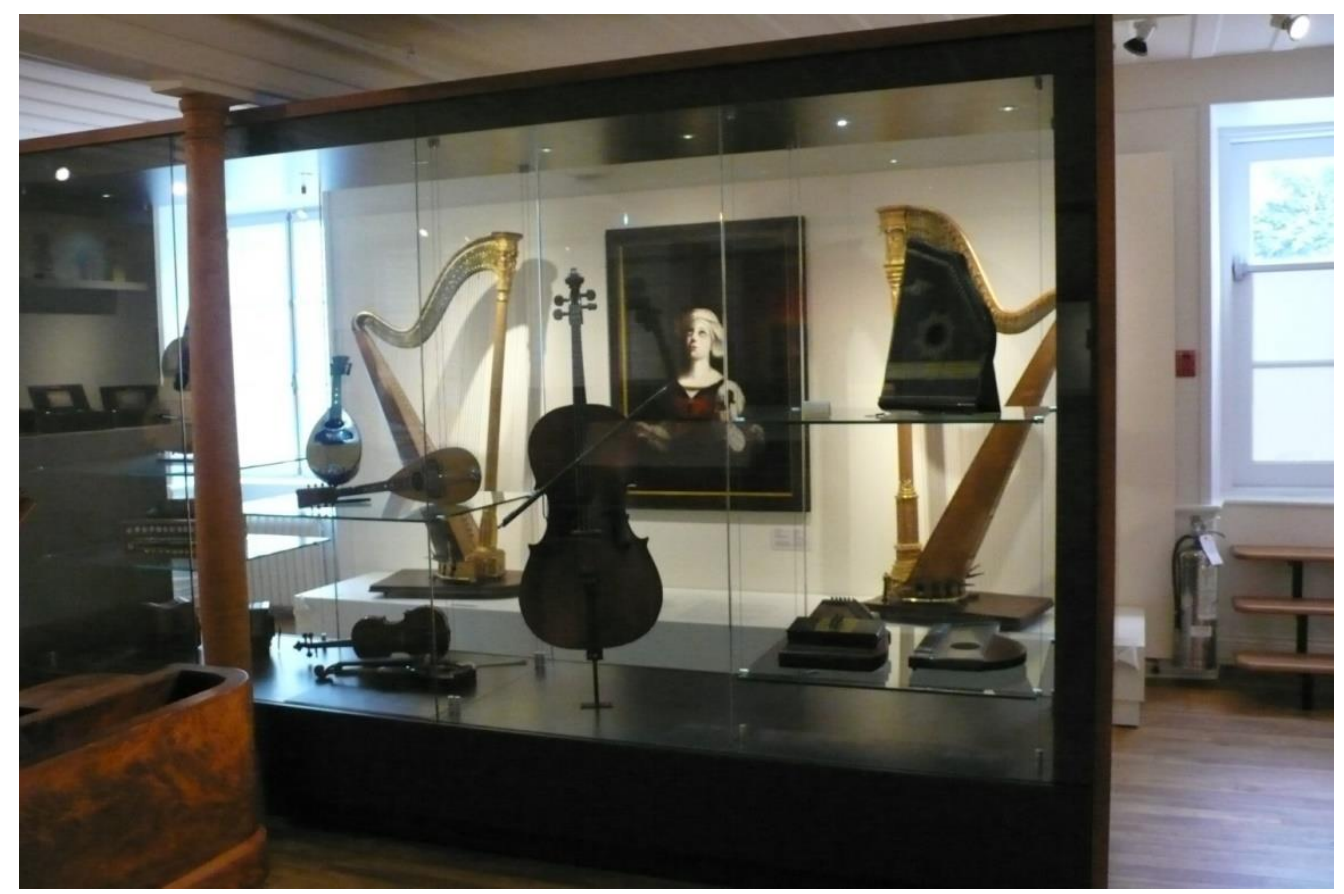

Fig. 2: Une partie de la salle « briller en société »

Les paroles des enfants sont citées de préférence in extenso en notes de bas de page afin de permettre au lecteur de mieux les saisir.

Ce sont trois salles du musée des Ursulines à Québec qui ont été présentées par ces enfants: «Au son de la cloche », « Briller en société » et «Le grand art de la broderie ».

Par exemple, la salle: «Briller en société » présente la place accordée à l'art dans l'éducation, à la sociabilité et à l'éducation au goût. Des dessins et peintures d'élèves, instruments de musique et travaux à l'aiguille sont exposés. Ces salles ont été décrites par Turgeon (2004) alors directrice et conservatrice du musée. 


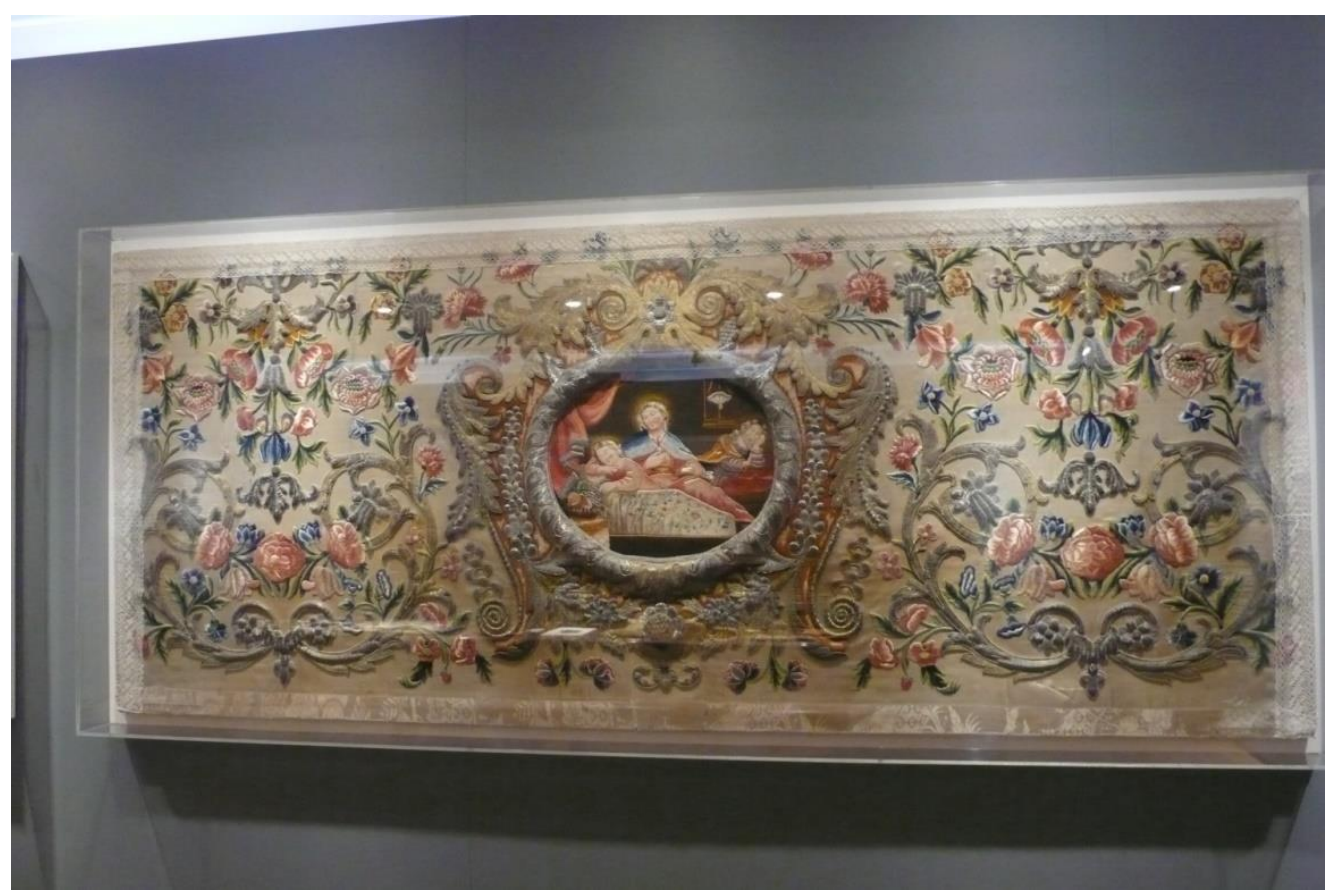

Fig. 3: Une des broderies installées dans la salle « grand art de la broderie »

\section{Appuis théoriques pour l'analyse des présentations des salles par les enfants}

\subsection{L'expérience selon la perspective du philosophe Dewey}

Cette recherche s'inspire des propos du philosophe Dewey (2006) considérant l'expérience comme une affaire d'«interactivité de l'organisme et de l'environnement», l'expérience est «behavorial». La perspective phénoménologique défend l'idée que l'être humain perçoit le monde avec son corps, qu'il agit et ressent par son intermédiaire. Dans le cas de la visite d'exposition, nous considérons que le visiteur réagit avec son corps en fonction des interactions avec l'exposition. Ses sens sont mis en action et l'aident à appréhender ce nouvel environnement. Face à l'insuffisance de l'intelligibilité pour saisir le monde, Dewey souligne l'enjeu de $l^{\prime}$ « ajustement sensible ${ }^{2} »$, et par la même occasion celui de l'expérience esthétique qui a été son

\footnotetext{
2 « La construction du monde social ne s'établit pas seulement dans son intelligibilité, mais aussi par un ajustement sensible »
} 
point de départ afin d'étudier l'expérience. Dans son ouvrage «Pour une éthique de la médiation », Caune met l'accent sur la place à accorder à l'expérience sensible et reprend les propos de Dewey considérant que celle-ci est le «fondement de la construction de l'être et de sa participation à la culture »(Caune, 2008: 48).

\subsection{Une expérience vécue dans l'espace de médiation de l'exposition}

L'expérience est mise en évidence à travers ce que « fabriquent $»^{3}$ les enfants quand ils visitent ou ici, quand ils présentent une salle d'exposition. Ce qui amène à s'interroger sur la manière de mettre en évidence cette expérience de visite en fonction de l'espace de médiation de l'exposition.

Le chercheur en muséologie, Silverstone (1998, p. 182) considère le musée de sciences en termes d'espaces dans lequel des objets et leur mise en scène sont offerts au public, proposant ainsi un «éventail de possibilités discursives ». Ces espaces sont qualifiés d'espaces discursifs avec lesquels les visiteurs établissent des relations contribuant à l'élaboration de leur démarche interprétative ${ }^{4}$. Il s'agit de l'espace de la rhétorique, du jeu (présentés ultérieurement) et celui de la performance, c'est-à-dire l'espace où les acteurs peuvent investir leur propre subjectivité, confronter leurs points de vue, procéder à des réajustements. Il nous a semblé audacieux de reprendre cette idée d'espaces discursifs dans le cas de musées d'histoire ou de civilisation.

\footnotetext{
${ }^{3}$ Expression empruntée à de Certeau, dans « Arts de faire », en 1993.

${ }^{4}$ Silverstone R., 1998, «Les espaces de la performance : musées, science et rhétorique de l'objet », pp. 175 à 188 , in : La mimésis, Hermès, $\mathrm{n}^{\circ} 22$.

Les espaces discursifs «dans lesquels les visiteurs participent à la construction du sens ». et (aux) diverses manières de le faire qui leur sont offertes »

On peut dire qu'au musée les visiteurs traversent ces espaces comme s'ils suivaient la trame d'un récit lorsqu'ils rencontrent (ou évitent) les différents objets exposés. En un mot, le musée, comme d'autres médias, propose un éventail d'engagements textuels possibles. Chacun d'entre eux se caractérise par un type distinct de réponse cognitive, affective, ou fondée sur l'expérience.
} 
De plus, nous avons pu observer lors de nos travaux de recherche (Martin, 2011) que les visiteurs-enfants vivaient aussi une expérience sensible, expression empruntée à Dewey et évoquée précédemment. D'où la prise en considération d'un espace discursif que nous avons nommé l'espace de l'esthétique avec lequel ces visiteurs sont en mesure d'établir des relations lors de la visite. Pour définir plus précisément l'expérience vécue avec cet espace, nous nous sommes inspirées des travaux de Caune (2008) qui invite à réfléchir sur les médiations qui sont en mesure de susciter une expérience artistique caractérisée par sa capacité d'influencer notre perception, de conditionner notre imaginaire, de mobiliser nos émotions et notre implication affective »(Caune, 2008: 65). Ce sont ces différentes dimensions que nous envisageons de prendre en considération pour décrire et analyser l'expérience vécue par les enfants avec cet espace de l'esthétique. Dans cet article, nous développerons principalement les relations établies par des enfants avec l'espace de la rhétorique et celui de l'esthétique.

\section{Expériences de la rhétorique vécues par les enfants}

L'étude de la relation des enfants avec l'espace de la rhétorique s'inspire des propos de Silverstone (1998, p. 183) sur la constitution de la rhétorique construite par le musée, à savoir «des objets, des commentaires et de plus en plus fréquemment d'images, d'installations [...]. Les relations établies par les enfants avec l'espace de la rhétorique donnent des indications sur leur manière d'aborder l'approche conceptuelle de l'exposition, qui inclut le propos de l'exposition, le titre de celle-ci et les notions abordées, ainsi que la cohérence d'ensemble. 


\subsection{La reconnaissance du propos de la salle}

À partir d'une vue d'ensemble de la salle d'exposition (« au son de la cloche »), l'enfant est capable de resituer le contexte. Il se projette dans la vie d'un enfant pensionnaire à partir de ce qu'il appelle « une reproduction » du lieu de vie et apprécie la qualité de vie de cette époque5.

\subsection{La présentation par les enfants des lieux et des objets du quotidien}

L'enfant E1 reconnaît la thématique traitée dans la salle en s'appuyant sur l'illustration sur le mur qui représente le dortoir, devant laquelle est positionnée une installation individuelle ; un lit, un meuble de chevet et le nécessaire pour la toilette, des livres. À partir de ces éléments, l'enfant reconstitue la vie d'une élève au dortoir tout en évoquant les similitudes avec la vie quotidienne de nos jours ${ }^{6}$. Elle se réfère à son propre monde et procède par analogie pour mieux comprendre la vie au dortoir (manuels ou livres de chevet, le nécessaire pour la toilette ou lavabo...).

Des enfants sont capables de se faire une idée de l'éducation autrefois à l'école des Ursulines à travers la présentation de certains objets. Par exemple, ce sont des images et des objets relatifs à la pratique sportive, tel un livre de dessins d'exercices physiques, qui permettent à une enfant d'interpréter ce que pouvait être l'éducation physique ${ }^{7}$ «cela ressemble à de l'éducation physique». Quant à une autre enfant, cela lui fait penser aux exercices cardio-vasculaires pratiqués de nos jours.

Différents objets concernant la musique sont repérés par des enfants et mobilisent leur sens de l'observation, qu'il s'agisse des instruments de musique, de métronome, de partitions, de

\footnotetext{
${ }^{5} \mathrm{E} 1$ : Là, dans cette salle-là, ça explique la rentrée scolaire, les choses comme ça. Donc ça c'est une reproduction des dortoirs, de comment était leur lit avec ce qu'il avaient à côté d'eux pour se laver les mains [...].

${ }^{6} \mathrm{E} 1$ : Ca c'est comme un peu leur lavabo. Il y a des photos et des livres de qu'est-ce qu'elles avaient, pour des manuels de comment vivre la vie ensemble.

${ }^{7} \mathrm{E} 2$ : Ça c'est bah, c'est un peu comme une médecine du sport, ils font des mouvements cardio-vasculaires, des choses comme ça. Ça ressemble à de l'éducation physique.
} 
photographies des élèves musiciennes, voire même de procédés pour accorder les instruments ${ }^{8}$. Concernant les instruments de musique exposés, des enfants sont en mesure d'en reconnaître et de les nommer (des harpes, du violoncelle, des violons, des guitares [... ], des accordéons, un accordéon [...], des grosses harpes). Elles en apprécient aussi leurs caractéristiques qui ne sont pas à sous-estimer comme le poids et à leur encombrement qui rendent d'autant plus contraignante leur manipulation: Et puis c'est gros, j'imagine que ça doit être lourd à traîner. Une autre enfant anticipe les difficultés supplémentaires pour la pratique d'un instrument telle la harpe. Ce sont aussi ses souvenirs de tourne-disque qui sont remémorés lorsqu'elle fait allusion à l'usage des disques de vinyle qu'elle a pu découvrir par ailleurs pour écouter de la musique ${ }^{9}$. Des enfants procèdent à des comparaisons entre les pianos d'autrefois et les pianos électriques d'aujourd'hui; elles prennent conscience de la rapidité de l'évolution de leur fabrication ${ }^{10}: E n$ peu de temps, ça a beaucoup évolué.

Leur sens de l'observation les conduit à explorer aussi les détails dans les écrits présentés à travers les livres ouverts dans la vitrine. Il est alors question de l'évolution de l'orthographe dans le temps: Nous au « règlement» on met un «t», c'est comme s'il y avait une faute mais c'est juste nous qui avons changé l'écriture ${ }^{11}$. Elle procède à une mise en relation temporelle, pour resituer ce document exposé dans le contexte d'une ancienne orthographe, différente de celle d'aujourd'hui.

${ }^{8} \mathrm{E} 3$ : Ça c'est des métronomes, donc des cahiers de musique, ça c'est des craies pour faire une portée. Ça c'est le son de la corde de la harpe, quand on l'accorde, ça fait ce son-là pour accorder son instrument. Ça c'est une photo des jeunes pensionnaires qui faisaient de la musique.

${ }^{9} \mathrm{E} 4$ : [...] ça, je ne sais pas trop ce que c'est, j'ai déjà vu ça, je pense que c'est... ça ressemble à où on mettait les disques de vinyle, pour faire de la musique.

${ }^{10}$ E3 : En regardant ce piano là et puis en regardant les pianos électriques qu'on a présentement, c'est vraiment... En peu de temps, ça a beaucoup évolué.

${ }^{11}$ E5 : Et puis j'ai remarqué, même si je le savais déjà, y a plusieurs mots qui ne s'écrivent pas comme nous. Nous au "règlement» on met un " $t$ », c'est comme s'il y avait une faute mais c'est juste nous qui avons changé l'écriture.

E6 : c'est pas la nouvelle orthographe, c'est pour eux la vieille orthographe. 
Ces jeunes «guides » reconnaissent les ustensiles de cuisine présentés dans la vitrine et leur fonction, comme les moules pour faire les gâteaux, la râpe à fromage, les bols à mélanger et le tablier de la cuisinière ${ }^{12}$.

\subsection{Les intentions pédagogiques comme éclairage sur l'éducation autrefois}

Il est possible de saisir l'émergence des intentions pédagogiques perçues par les enfants en fonction de certains objets exposés. Devant plusieurs bustes, dont celui de Frédéric Chopin, une enfant perçoit cette sculpture du compositeur comme une source d'inspiration pour les élèves en cours d'apprentissage de la musique ${ }^{13}$.

L'attention d'une enfant est centrée sur la «boîte à récompenses » qui est un appui pour comprendre le système éducatif, basé sur les récompenses et, selon elle, aussi sur les punitions ${ }^{14}$. Les efforts fournis pour atteindre le niveau attendu dans les apprentissages sont validés par l'obtention d'un diplôme, constat fait par une des enfants ${ }^{15}$.

\subsection{La perception des objets et des personnages en lien avec la religion et la Communauté des}

\section{Ursulines, la dimension historique du lieu}

Le musée se situe actuellement sur l'emplacement de la maison de Mme Peltrie. Une enfant précise que, suite à un incendie, les religieuses ont habité cette maison. En tant qu'élèves de l'école des Ursulines, les enfants « guides » ont déjà une connaissance des personnages principaux qui ont contribué à la construction du Monastère des Ursulines et de l'école à Québec: Marie de

\footnotetext{
${ }^{12}$ E2 : C'est ça. Là y a des moules pour faire des gâteaux ou des trucs comme ça, en plusieurs formes. Une râpe à fromage et puis des bols à mélanger. Et puis peut-être un costume de cuisinière.

${ }^{13}$ E6 : Bah je remarque Frédéric Chopin, c'est plusieurs artistes qui sont représentés et puis je pense que c'est une belle inspiration pour les jeunes musiciens.

${ }^{14}$ E2 : Et puis aussi, ce que j'ai oublié de dire, c'est que la petite boîte, une boîte à récompenses donc ils peuvent chercher une récompense quand ils font des choses correctes.

${ }^{15}$ E5 : ça c'est un diplôme d'honneur, probablement qu'une fille a passé, probablement un niveau de musique et puis elle a reçu un diplôme.
} 
l'Incarnation pour la fondation du Monastère et Mme de la Peltrie pour son soutien financier. Les enfants ont conscience du rôle de Marie de l'Incarnation puisqu'elle est à l'origine de la construction de leur école. Il est fort probable que ces jeunes « guides » aient été sensibilisés à leur école sur l'origine de la fondation de leur institution ${ }^{16}$ et aux modalités d'éducation à cette époque. Les raisons de la venue de Marie de l'Incarnation au Québec sont expliquées ainsi par des enfants: "Parce qu'elle a reçu un message de Dieu pour fonder une école au Canada». Cette enfant a gardé en mémoire cet événement religieux qui a marqué l'histoire de leur institution et l'histoire de leur pays.

Une enfante tente d'expliquer le devenir des pensionnaires ${ }^{17}$, soit religieuse, soit femme à la maison, et elle précise que ce sont les seules alternatives : Et puis je crois que c'était pas mal les deux seules options.

Devant la chasuble de prêtre brodée, des enfants reconnaissent l'habit du prêtre utilisé pour les cérémonies religieuses. Elles associent la qualité et la richesse du travail de broderie à l'importance du contexte de son usage, à savoir des célébrations très « importantes », au moment d'occasions exceptionnelles ${ }^{18}$.

\subsection{Le musée, un lieu de découvertes, d'accès aux connaissances}

Si visiter un musée permet d'accéder à des connaissances, des enfants n'hésitent pas à mentionner qu'ils savent déjà des choses. Une enfant ajoute que ce n'est pas une découverte pour

\footnotetext{
${ }^{16}$ E2 : Marie de l'Incarnation, c'est la fondatrice de notre école qui a bâti notre école. [...] Mais la plupart du temps, on la reconnaissait avec son habit de religieuse.

E1 : C'est la fondatrice avec Marie de l'Incarnation. C'est elle qui a financé en même temps.

${ }^{17}$ E1 : Bah elles pouvaient être religieuses si elles décidaient de rester là. Elles pouvaient devenir juste une femme à la maison, puisque dans ce temps-là c'est ce que toutes les femmes faisaient. Et puis je crois que c'était pas mal les deux seules options.

${ }^{18}$ E1 : je crois que c'est peut-être un habit de prêtre, lors des célébrations et des plus grandes célébrations, des messes très importantes.
} 
elle: « Et puis j'ai remarqué, même si je le savais déjà, y a plusieurs mots qui ne s'écrivent pas comme nous ».).

\section{Expériences ludiques vécues par les enfants}

Dans l'espace discursif du jeu, les visiteurs «se lancent » dans cet espace du jeu, quittent le monde réel pour s'approprier le monde synthétique de l'exposition, tout en minimisant la prise de risque (Silverstone, 1998: 185). Cette expérience ludique s'explique par le changement de cadres d'interprétation, par le passage du cadre primaire (cadre fictif du monde de l'exposition représenté) au cadre secondaire (réel), si l'on se réfère à Goffman (1991) et Brougère (2005).

Si l'espace du jeu est surtout offert dans les institutions muséales de sciences, il est plus rarement présent dans les musées d'histoire, car les enfants n'ont pas la possibilité de s'approprier les objets en les manipulant. L'expérience ludique se rattache ici à la mise en situation de «guide » présentant une salle d'exposition. Une enfant procède à une mise en scène lorsqu'elle évoque une «anecdote». Elle relate un souvenir de sa voisine, ancienne pensionnaire qui lui avait témoigné de son engouement pour la broderie au point qu'on aurait pu croire que ses parents avaient contribué à sa confection ${ }^{19}$.

\section{L'expérience de l'esthétique vécue par les enfants}

Pour analyser l'expérience esthétique vécue chez ces enfants «guides», nous prendrons en considération leur perception sur les objets et les personnages historiques, voire éventuellement la mise en scène, leur sensibilité esthétique qui englobe leur jugement de goût (appréciation),

${ }^{19}$ E3 : en fait ma voisine, elle est allée aux Ursulines (demi-pensionnaire). Et puis je me souviens qu'elle, il y avait des travaux qu'ils lui demandaient de faire comme admettons faire de la broderie ou de la couture ou des choses comme ça. [...]. Et puis elle, elle aimait tellement faire ça qu'elle en faisait encore plus et puis là les sœurs lui demandaient " est-ce que c'est votre mère ou quelque chose comme ça qui le fait » parce que tu sais, à en faire plus et puis c'est ça. Petite anecdote. 
leurs préférences, leur implication affective, et les émotions mobilisées, selon l'éclairage apporté par Caune.

\subsection{Sensibilité esthétique des enfants à travers leur jugement de goût}

Nous faisons part ici de la perception des enfants sur une sélection d'objets qu'elles ont choisi de présenter. Des enfants sont sensibles à la beauté des instruments de musique exposés dans la salle «Briller en société », à la fois pour la qualité de leur fabrication et pour le son qu'ils peuvent produire: je trouve ça vraiment beau, je trouve que ça fait un beau son, les cordes je trouve ça vraiment impressionnant. Une autre enfant insiste sur la place du piano dans l'éducation musicale. Elle constate que les pianos exposés sont nombreux et en déduit que le piano devait être l'instrument principalement joué par les élèves. [...] la plupart des jeunes faisaient du piano. C'est le centre de la musique, c'est un peu ce qui est arrivé en premier. Au point de considérer le piano comme le $« r o i »$ des instruments ${ }^{20}$. Les enfants reconnaissent les enjeux de la place de la musique dans l'éducation ${ }^{21}:[. .$.$] je trouve ça fascinant à quel point la$ musique est restée présente dans l'école et puis à quel point l'art a pris une grande place dans la vie des jeunes.

Des enfants sont sensibles à la création artistique. Devant les parements d'autels brodés notamment au fil d'or et en relief, des enfants apprécient la qualité de confection: Je pense que c'est de la broderie, des fleurs cousues peut-être. Ça ressemble à des fils de couture. Ou selon

\footnotetext{
${ }^{20}$ La plupart des jeunes faisaient du piano. C'est le centre de la musique, c'est un peu ce qui est arrivé en premier Et puis il est très populaire, les jeunes jouent beaucoup de piano, c'est pour ça que c'est un peu le roi, parce qu'il y en a beaucoup.

${ }^{21}$ Mais moi j'ai décidé de présenter de cette salle-là parce que je trouve ça fascinant à quel point la musique est restée présente dans l'école et puis à quel point l'art a pris une grande place dans la vie des jeunes.
} 
une autre enfant: les fleurs sont très belles ${ }^{22}$.

L'état de conservation des objets anciens n'échappe pas certaines «guides » ${ }^{23}$ : je trouve ça impressionnant qu'ils aient gardé et puis que, qu'ils aient gardé ces choses-là. Ces objets exposés sont resitués dans le contexte du musée et de conservation, voire de transmission puisqu'émerge la prise de conscience de cet accès à l'histoire de leur institution, de leur pays de leur place en tant qu'élève au sein de cette institution: "C'est impressionnant qu'il y ait encore ces objets-là qui sont quand même importants pour l'histoire ». Tardif s'interrogeant sur le «pour quoi faire » du musée d'éducation mentionne qu'on ressent tous, un jour, le besoin de connaître ses origines puis de les transmettre à ses descendants. Il rappelle le devoir de mémoire.

\subsection{L'implication affective}

Le contexte du pensionnat recréé par la salle «au son de la cloche » suscite chez les enfants l'imagination pour se représenter la vie au pensionnat et son ambiance du fait de l'éloignement des parents. Des enfants ont de l'empathie pour les pensionnaires qui devaient ressentir un tiraillement à être séparées de leurs parents ${ }^{24}$ : Je pense que la première journée était très difficile, parce que tu venais de quitter tes parents [...] Le fait de rester au pensionnat encadrée par le personnel de l'institution et sans rentrer à la maison le soir semble rendre plus contraignante la vie d'enfant avec les règles établies par l'institution. Cela contribue ainsi au caractère «strict » évoqué par l'une des « enfants-guides » : et puis quand même, ils sont stricts, il faut que tu t’habitues, mais je pense qu'après ça, quand on est habitué, ça devient naturel. Elle insiste sur le début du pensionnat qui devait être difficile et constituer en quelque sorte un cap à

\footnotetext{
${ }^{22}$ Ça ressort et puis c'est quand même très joli, on voit comme si quelque chose sortait du cadre. Ils ont bien encadré la photo et puis les fleurs sont très belles.

${ }^{23}$ « C'est impressionnant qu'il y ait encore ces objets-là qui sont quand même importants pour l'histoire, je trouve ça impressionnant qu'ils aient gardé et puis que, qu'ils aient gardé ces choses-là ».

${ }^{24}$ Je pense que la première journée était très difficile, parce que tu venais de quitter tes parents et puis quand même, ils sont stricts, il faut que tu t'habitues mais je pense qu'après ça, quand on est habitué, ça devient naturel.
} 
dépasser.

Parmi ces jeunes «guides », certaines expriment leur appréciation sur la démarche d'éducation des sœurs converses surnommées «matantes » ${ }^{25}$. L'une d'elles imagine le rôle de ces religieuses dont la présence compense d'une certaine façon l'absence des parents en gâtant les enfants avec des gâteaux ou en étant dispensatrice de bons conseils ou à l'écoute des confidences $^{26}$. Cet enfant se projette comme pensionnaire et accorderait sa confiance à une sœur converse pour le soutien qu'elle peut apporter, et l'appui face aux incertitudes. La sœur converse est perçue comme une personne à l'écoute, avec qui parler. C'est une manière de percevoir la dimension intime ou affective de la vie de pensionnaire; cet enfant perçoit les interactions possibles avec son entourage d'autant plus que celui-ci peut être une oreille attentive à ses interrogations.

Le musée expose des tableaux qui représentent les portraits des personnages principaux de la fondation du monastère des Ursulines à Québec, à savoir Marie de l'Incarnation, religieuse Ursuline de Tours et Madame de la Peltrie pour le soutien financier de leur mission. Celle-ci a aussi fait la traversée de l'Atlantique au 17e siècle, témoignant ainsi de son engagement dans la mission d'évangélisation. Des enfants ont conscience du rôle de Mme de la Peltrie auprès de Marie de l'Incarnation et de son engagement financier dans cette mission. L'une d'elles l'exprime ainsi: mais je pense qu'elle a été une personne importante pour Marie de l'Incarnation et qui a un peu changé sa vie [...]. Cet enfant devine les enjeux de cette aventure et des liens profonds qui devaient unir ces deux personnages pour mener à bien leur mission,

${ }^{25}$ E6 : elles pouvaient être strictes mais c'est pas parce que t'es stricte que y a pas personne qui ne doit pas être gentil nécessairement avec. Donc elle était peut-être stricte sauf qu'elle pouvait permettre des choses, s'ils faisaient ça.

${ }_{26}^{26}$ "parce que admettons que de fait, y a des choses que tu sais pas et puis, pas que tu veuilles, tu as quelqu'un de confiance aussi, de savoir qu'il y a quelqu'un d'autre qui t'appuies ou quelque chose comme ça, ça devait sûrement être plus fun de pouvoir avoir quelqu'un avec qui parler». 
entre autres la construction de leur école ${ }^{27}$.

À propos des méthodes d'enseignement, le système de punitions et de tableaux d'honneur fait réagir les enfants. L'une d'elles aborde la motivation suscitée chez les élèves pour être bien classée et éviter les punitions ${ }^{28}$ : t'avais pas le goût d'être en bas. Le classement étant visible par tous, cela devait motiver les enfants à ne pas être en dernière position, et sous-entendu donc créer une stimulation pour atteindre les premières places.

\subsection{Les émotions mobilisées}

Face à la présentation d'un couvert présenté sur une table de réfectoire, une enfant s'émerveille et prend conscience à la fois du rôle de transmission du musée et de la découverte de sa propre histoire, celle d'élève de l'école des Ursulines: Y a des anciennes étudiantes du pensionnat qui ont mangé probablement dans cette assiette-là et puis je trouve ça quand même impressionnant.

L'attitude des enfants découvrant ainsi leur histoire d'élève révèle leur situation d'héritière. Nous pouvons supposer que ces fillettes prenant conscience de leur héritage (objets historiques de l'éducation des Ursulines) éprouvent de la fierté si l'on en croit les propos de Tardif (2006: $147)^{29}$ sur les enjeux de la transmission du patrimoine dans le cas des musées de l'éducation. À travers la découverte de leur patrimoine, les héritiers se reconnaissent en leurs prédécesseurs et ils saisissent d'autant mieux les valeurs transmises. Ils en tirent une « fierté certaine ».

\section{Les apports de cette recherche exploratoire}

\footnotetext{
${ }^{27}$ Mais je pense qu'elle a été une personne importante pour Marie de l'Incarnation et qui a un peu changé sa vie parce que aussi, si elle n'avait pas été là pour Marie, elle n'aurait pas pu financer toutes ces choses-là et bien notre école n'aurait peut-être pas eu lieu, en fait.

${ }^{28}$ E6 : et puis comme t'avais des punitions si tu n'étais pas très bien classée dans le tableau d'honneur, puis t'avais pas le goût d'être en bas.

29 «En accueillant ce patrimoine, ces héritiers reconnaissent leurs prédécesseurs et se reconnaissent en eux. Ils en tirent une fierté certaine. Ils comprennent ainsi un peu mieux qui ils sont, quelles sont leurs valeurs » (Tardif, 2006 : 147).
} 
À la sortie de l'exposition, un schéma récapitule les différentes expériences, de la rhétorique, du jeu et de l'esthétique vécus par des enfants lors de leurs présentations de salles d'exposition du musée des Ursulines à Québec, hors du temps scolaire.

Cette recherche exploratoire a pu mettre en évidence différentes expériences vécues par les enfants « guides ». Ce sont leurs relations avec l'espace de la rhétorique (manière d'appréhender l'éducation des jeunes filles), avec l'espace du jeu (dans le rôle de guide) et avec l'espace de l'esthétique (empathie pour les pensionnaires, appréciation de la conservation des objets du musée ...) qui ont été mises au jour.

Ces enfants « guides » s'appuient sur leur sens de l'observation pour interpréter et elles trouvent des réponses à leur questionnement sur la vie des pensionnaires par induction et par comparaison avec leur vie d'élève aujourd'hui. Les objets qu'elles présentent et leurs usages sont resitués dans le contexte des pensionnaires. C'est ainsi qu'elles se font une idée de la vie de pensionnaire au 19e siècle, des intentions pédagogiques, et par conséquent une représentation de l'éducation dispensée à l'école des Ursulines à cette époque. Leur imagination est aussi à l'œuvre quand elles tentent de se représenter la vie des pensionnaires, de même pour témoigner de leur empathie pour ces élèves. Cela peut aussi les conduire à porter intérêt au média-exposition à travers notamment l'appréciation de la qualité de la conservation et les objectifs de sensibilisation à l'histoire de leur école. C'est aussi le rôle du conservateur ou conservatrice qui est sous-entendu à travers les propos des enfants. Pour transmettre ce patrimoine, précise Tardif (2006: 147), il faut «identifier, inventorier, repérer ce qui a du sens, ce qui mérite d'être conservé, pour ensuite le diffuser et le faire connaître. [...]. Conserver le patrimoine et le mettre en valeur n'est pas en soi un geste égoïste, mais un acte de générosité envers les générations futures». En présentant l'exposition, ces enfants « guides », découvrent ce qui constitue la «mémoire » de leur école et 
prennent conscience leur histoire.

\section{Bibliographie}

Brougères, G. (2005). Jouer/apprendre. Édition Economica, (Coll. éducation).

Caune, J. (2008). Pour une éthique de la médiation: Le sens des pratiques culturelles (Coll. Communication, médias et sociétés). Grenoble: PUG.

de Certeau, M. (1990, 2005). Arts de faire : Vol. 1. L'invention du quotidien, Paris: Éditions Folio essais.

Dewey, J. (2006). Les æuvres philosophiques: Vol. 3. L'art comme expérience ( J. -P. Cometti, C. Domino, F. Gaspari, \& al., Trad.). Pau: Presses de l'Université de Pau. (Euvre originale publiée en 1934).

Gaulupeau, Y. (2006). L’éducation, un objet de musée. In Anik Meunier (ss dir.). Patrimoine scolaire, sa sauvegarde et sa valorisation. Québec, QC: Éditions Marquis. P 27 - 33.

Goffman, E. (1991). Les cadres de l'expérience (I. Joseph, M. Dartevelle, \& P. Joseph, Trad.). Paris : Éditions de Minuit. (CEuvre originale publiée en 1974).

Martin, T. (2011). L'expérience de visite des enfants en musées de sciences dans le cadre des loisirs. Logiques d'interprétation et enjeux d'un dispositif communicationnel. Thèse de doctorat en Sciences de l'Information et de la Communication. Université Lille 3, Lille. En ligne http://tel.archives-ouvertes.fr/tel-00783563

Martin, T. (2012). Les logiques d'interprétation des enfants selon leur expérience de visite dans les musées de sciences et dans le cadre des loisirs. Communication, 30(2). Doi: 10.4000/communication.3598. En ligne:

http://communication.revues.org/index3598.html

Poulot, D. (2001). Patrimoine et musées. L'institution de la culture. Paris: Hachette 
Poulot, D. (2015, mars). Les rôles nationaux des musées d'art. Communication présentée au Colloque international : Frontières du patrimoine. Circulation des savoirs, des objets et œuvres d'art. Paris: Institut national d'histoire de l'art.

Silverstone, R. (1998). Les espaces de la performance : Musées, science et rhétorique de l'objet. Hermès, 22, 175-188.

Tardif, J-C. (2006). L'avenir du patrimoine scolaire. In A. Meunier (ss dir.). Patrimoine scolaire, sa sauvegarde et sa valorisation (pp. 145-150). Québec, QC: Éditions Marquis.

Turgeon, C. (2004). Le musée des Ursulines de Québec: Art, foi et culture. Québec, QC: Bibliothèque Nationale du Québec, Monastère des Ursulines de Québec. 